\title{
Controle preditivo baseado em modelo com retroalimentação aplicado no conversor boost com incertezas *
}

\author{
Rosana C. B. Rego* Marcus V. S. Costa** \\ * Departamento de Engenharia e Tecnologia, Universidade Federal \\ Rural do Semi-Árido, RN, (e-mail: rosana.rego@ufersa.edu.br). \\ ** Departamento de Engenharia, Universidade Federal Rural do \\ Semi-Árido, RN, (e-mail: marcus.costa@ufersa.edu.br)
}

\begin{abstract}
This paper proposes an off-line formulation for predictive control based on constrained robust model (MPC) for uncertain systems based on a quasi-min-max algorithm with linear matrix inequality (LMI). The approach involves an off-line design of a robust state observer. The proposed off-line MPC is applied to the three-state switching cell (3SSC) boost converter. A comparison is made between online and off-line performance of the algorithm. The results showed that it is possible the off-line implementation of the output feedback MPC and the simulation obtained good results.

Resumo: Este artigo propõe uma formulação off-line para o controle preditivo baseado em modelo (MPC) robusto com restrição para sistemas incertos baseados em um algoritmo quasimin-max com desigualdades matriciais lineares (LMI). A abordagem envolve um design off-line de um observador de estado robusto. O MPC off-line proposto é aplicado ao conversor boost de célula de comutação de três estados (CCTE). Uma comparação é realizada entre o desempenho online e off-line do algoritmo. Os resultados mostraram que é possível a implementação off-line do MPC com retroalimentação e a simulação obteve bons resultados.
\end{abstract}

Keywords: Model predictive control; Boost converter; Linear Matrix Inequalities; Uncertainties. Palavras-chaves:Controle preditivo baseado em modelo; Conversor boost; Desigualdades matriciais lineares; Incertezas.

\section{INTRODUÇÃO}

Os conversores DC-DC são bastante utilizados nos sistemas de fornecimento de energia, eles são considerados a maneira mais eficiente de implementar atuadores para sistemas eletromecânicos, principalmente nas estruturas de eletrônica de potência (Olalla et al., 2012). O objetivo dos conversores DC-DC é fornecer um saída de tensão DC mesmo quando sujeito a carga ou variações de tensão de entrada (Guldemir, 2011).

Um dos conversores DC-DC de interesse, principalmente na areá de eletrônica de potência são os conversores boost (Costa et al., 2017). Um conversor DC-DC boost tem a capacidade de elevar uma dada tensão DC. Ele possui uma topologia simplificada, porém apresenta algumas singularidades em sua modelagem, tais como as variações de resistência de carga, tensão de entrada e os efeitos da fase não mínima como apresentado por Linares-Flores et al. (2014) e Cheng et al. (2017). Realizar o controle desses conversores é considerado uma tarefa complicada pelo fato de o modelo ser de fase não mínima (Amirifar, 2005; Costa, 2017).

\footnotetext{
^ O presente trabalho foi realizado com apoio da Coordenação de Aperfeiçoamento de Pessoal de Nível Superior - Brasil (CAPES) Código de Financiamento 001.
}

O controle preditivo baseado em modelo $(M P C$ - model predictive control) mostrou ser um tipo de controle bastante robusto na maioria das aplicações, tais como em conversores estáticos e em dispositivos de acionamentos elétricos (Camacho e Alba, 2013; Costa, 2017). As principais causas desse controle é que ele pode ser tanto aplicado a sistemas monovariáveis como multivariáveis linear ou não-linear (Camacho e Alba, 2013; Aguirre et al., 2007; Costa, 2017).

O MPC para sistemas lineares incertos foi extensivamente estudado na última década. Foi proposto com base no conceito de conjuntos invariantes elipsoidais (Kothare et al., 1996). Wan e Kothare (2002) propuseram uma formulação off-line para o MPC-LMI iterativo proposto por Kothare et al. (1996). Desta forma, tornou-se possível garantir a estabilidade do modelo restrito utilizando apenas um ganho estático, simplesmente armazenando-o em um conjunto de ganhos, chamado de look-up table.

Além disso, os trabalhos que utilizam o MPC off-line com modelos incertos aumentaram nos últimos anos, conforme Paulson et al. (2017); Lorenzen et al. (2017); Zheng et al. (2018); Moradi et al. (2018); Hu e Ding (2019).

Para mais, Longge e Yan (2017) propõem uma nova estratégia off-line MPC com incerteza politópica baseado nas elipsóides de estabilidade usando otimização LMI. 
Ping (2017) propõe um parâmetro de escalonamento para feedback de saída do MPC-LMI robusto, aplicando um look-up table off-line em um modelo de reator (CSTR). Hu e Ding (2019) propuseram uma generalização do trabalho de Wan e Kothare (2002) e Wan e Kothare (2003) para o caso quando há estado não mensurável e com perturbação.

Desta forma, este trabalho utilizou o conceito de elipsoides de estabilidade, definindo um conjunto de pontos fixados na elipsoide, como proposto por Wan e Kothare (2002) e Wan e Kothare (2003). Usando a técnica MPC com feedback de saída proposta por Park et al. (2011) e Kim e Lee (2017) propusemos uma formulação off-line para o algoritmo quasi-min-max baseado na técnica MPC com feedback de saída. A formulação proposta é aplicada no conversor boost com célula de comutação de três estados (CCTE) com incertezas (Costa, 2017). As incertezas do conversor consideradas são as variações de resistência de carga e tensão de entrada.

O artigo está organizado da seguinte forma. Primeiro, na seção 2, é apresentada a modelagem matemática do conversor boost e a estratégia de controle. Na seção 3, o problema é apresentado e alguns conceitos básicos sobre o MPC são relembrados. Na seção 4, o algoritmo off-line é mostrado. Na seção 5, o exemplo numérico é apresentado com análise comparativa entre o MPC online e off-line. Finalmente, na seção 6 , discutimos as conclusões do estudo. Notação. $O$ símbolo * é usado em algumas expressões matriciais para induzir uma estrutura simétrica. Por exemplo

$$
\left[\begin{array}{ll}
Q & * \\
S & P
\end{array}\right]=\left[\begin{array}{ll}
Q & S^{T} \\
S & P
\end{array}\right]
$$

\section{CONVERSOR BOOST}

A Figura 1 mostra o conversor boost usado, o mesmo porposto em Bascopé e Barbi (2000); Costa (2012, 2017),

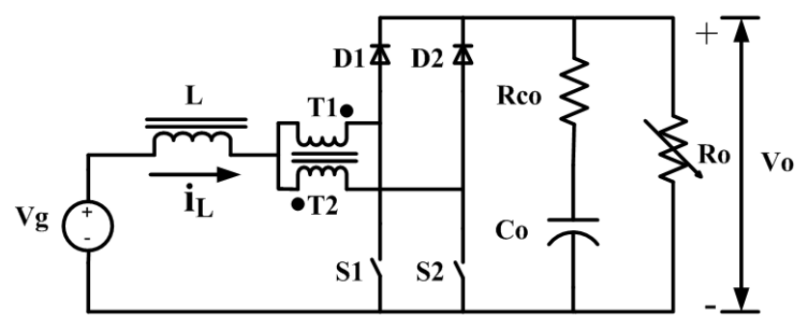

Figura 1. Conversor boost com célula de comutação de três estados.

Os parâmetros utilizados para o conversor podem ser vistos na tabela 1, os mesmos usados por Rego et al. (2018).

\subsection{Modelagem matemática}

As expressões no espaço de estados $A_{t}, B_{t}, C_{t}$ and $D_{t}$ operando no modo de condução contínua (CCM) de acordo com Middlebrook e Cuk (1976) são:

$$
\begin{aligned}
& \dot{x}=A_{t}(t) x+B_{t}(t) u, \\
& y=C_{t}(t) x+D_{t}(t) u,
\end{aligned}
$$

Tabela 1. Parâmetros do conversor boost

\begin{tabular}{ll}
\hline Parâmetros & Valores \\
\hline Tensão de Entrada $\left(V_{g}\right)$ & $26-36[\mathrm{~V}]$ \\
\hline Tensão de saída $\left(V_{o}\right)$ & $48[\mathrm{~V}]$ \\
\hline$D_{\text {cycle Ciclo de Trabalho }}$ & $0.25-0.46$ \\
\hline Frequência de chaveamento $\left(f_{s}\right)$ & $22[\mathrm{kHz}]$ \\
\hline Indutor de Filtro $(\mathrm{L})$ & $36[\mu H]$ \\
\hline Resistência Indutiva $\left(R_{L}\right)$ & $0[\Omega]$ \\
\hline Capacitor de saída $\left(C_{o}\right)$ & $4400[\mu F]$ \\
\hline Resistência serie $\left(R_{c o}\right)$ & $26.7[m \Omega]$ \\
\hline Carga $\left(R_{o}\right)$ & $2.034-6.06[\Omega]$ \\
\hline Potência de saída & $380-1000[\mathrm{~W}]$ \\
\hline Passo de simulação & $1 \mu \mathrm{s}$ \\
\hline
\end{tabular}

onde,

$$
\begin{gathered}
A_{t}=\left[\begin{array}{cc}
-\frac{\left(1-D_{\text {cycle }}\right)\left(R_{c o} \| R_{o}\right)}{L}-\frac{\left(1-D_{\text {cycle }}\right) R_{o}}{L\left(R_{c o}+R_{o}\right)} \\
\frac{\left(1-D_{c y c l e}\right) R_{o}}{C_{o}\left(R_{c o}+R_{o}\right)} & -\frac{1}{C_{o}\left(R_{c o}+R o\right)}
\end{array}\right], \\
B_{t}=\frac{V_{g}}{R^{\prime}}\left[\begin{array}{c}
\frac{R_{o}}{L} \frac{\left(1-D_{c y c l e}\right) R_{o}+R_{c o}}{R_{o}+R_{c o}} \\
-\frac{R_{o}}{R_{o}+R_{c o}}
\end{array}\right] \\
C_{t}=\left[\begin{array}{c}
\left(1-D_{c y c l e}\right)\left(R_{c o} \| R_{o}\right) \\
R_{c o}+R_{o}
\end{array}\right] \\
D_{t}=-V_{g} \frac{R_{c o} \| R_{o}}{R^{\prime}} .
\end{gathered}
$$

em que $R^{\prime}=\left(1-D_{\text {cycle }}\right)^{2} R_{o}+D_{\text {cycle }}\left(1-D_{\text {cycle }}\right)\left(R_{\text {co }} \| R_{o}\right)$, $x=\left[i_{L} V_{c}\right]^{T}$ onde $i_{L}$ é a corrente no indutor, $V_{c}$ é a tensão no capacitor, $u$ é o sinal de controle, $D_{\text {cycle }}$ é o ciclo de trabalho e $y=V_{o}, V_{o}$ é a tensão de saída.

As incertezas do modelo podem ser definidas por (Costa et al., 2017; Costa, 2017):

$$
\begin{aligned}
& R_{o}=f(\text { Pot })=\frac{V_{o}^{2}}{\text { Pot }} \quad \text { Pot } \in[3801000], \\
& D_{\text {cycle }}=f\left(V_{g}\right)=1-\frac{V_{g}}{V_{o}} \quad V_{g} \in[2636] .
\end{aligned}
$$

\subsection{Estratégia de controle}

Para facilitar a análise, $A_{t}, B_{t}, C_{t}$ e $D_{t}$ são discretizados conforme Costa (2017). A Figura 2 mostra o diagrama de blocos proposto neste trabalho para obter o ganho do controle para o conversor.

As expressões do modelo são dadas por:

$$
\begin{gathered}
x(k+1)=A x(k)+B u(k) \\
\hat{x}(k+1)=A \hat{x}(k)+B u(k)+L_{o}(y(k)-\hat{y}(k)) \\
e(k)=r(k)-y(k) \\
v(k+1)=e(k) h+g v(k) \\
y(k)=C x(k)+D u(k) \\
\hat{y}(k)=C \hat{x}(k)+D u(k) \\
u(k)=-K \hat{x}(k)+K_{i} v(k)
\end{gathered}
$$

onde $A, B, C$, e $D$ são as matrizes do sistema discretizadas, $g, h$ são as matrizes que correspondem ao grau de liberdade da ação integral. $\hat{x}(k)$ é o estado observado e $L_{o}$ é o ganho do observado. $K_{i}$ e $K$ são respectivamente o ganho de ação integral e ganho do controlador MPC. A próxima seção descreve o procedimento para obter os ganhos. 


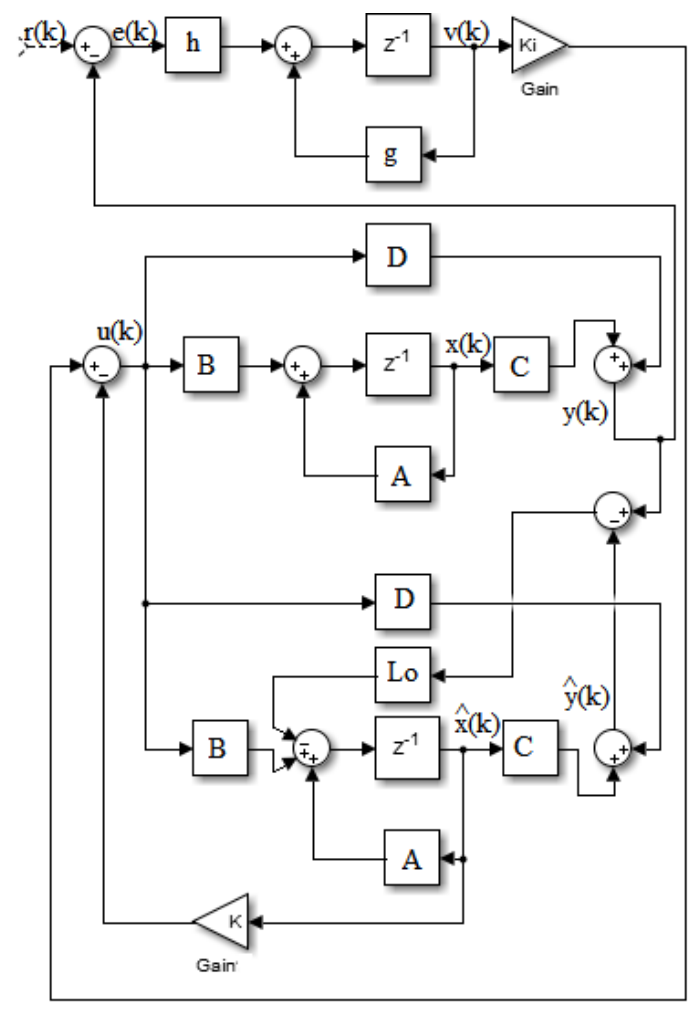

Figura 2. Diagrama de blocos.

\section{MPC COM REOTROALIMENTAÇÃO NA SAÍDA}

A técnica de controle MPC quasi-min-max para sistemas incertos consistem em minimizar uma função objetivo tal que,

com,

$$
\min _{u(k+i)} \max _{|A(p(k+i)) B(p(k+i))| \in \Omega, i \geq 0} J^{\infty}(k)
$$

$$
\begin{gathered}
J^{\infty}(k)= \\
+\hat{x}(k \mid k)^{T} \Psi \hat{x}(k \mid k)+u(k \mid k)^{T} R u(k \mid k) \\
+\sum_{i=1}^{\infty}\left(\hat{x}(k+i \mid k)^{T} \Psi \hat{x}(k+i \mid k)\right. \\
\left.+u(k+i \mid k)^{T} R u(k+i \mid k)\right) \\
<\zeta(k) .
\end{gathered}
$$

em que $p(k)$ é o parâmetro incerto, $\hat{x}$ é o vetor de estados observados, $u(k)$ é o vetor do sinal de controle, $y(k)$ é a saída do sistema, e $R, \Psi$ são matrizes pesos para a entrada e saída do sistema respectivamente. $\zeta(k)$ é o limite superior da função objetivo. As matrizes $[A(p(k)) \mid B(p(k))]$ pertencem a um politopo convexo definido por Wada et al. (2006),

$$
\begin{gathered}
{[A(p(k)) \mid B(p(k))] \in} \\
C o\left\{\left[A_{1}, B_{1}\right],\left[A_{2}, B_{2}\right], \cdots\left[A_{j}, B_{j}\right]\right\},
\end{gathered}
$$

onde $C o\{\cdot\}$ denota o casco convexo e $[A j, B j]$ são vértices de um conjunto politópico.

Considerando o fato de que os estados do sistema (1) são imensuráveis, será adotado o seguinte observador de estado para estimar os estados incertos do sistema.

$$
\begin{gathered}
\hat{x}(k+1)=A(p(k)) \hat{x}(k)+B(p(k)) u(k) \\
+L_{o}(y(k)-C(p(k)) \hat{x}(k)),
\end{gathered}
$$

em que $\hat{x}(k) \in \mathbb{R}^{n_{x}}$ é o estado estimado de $x(k)$, e $L_{o}$ é o ganho do observador.

Portanto, a formulação MPC é dada pelas seguintes LMIs (Park et al., 2011; Kim e Lee, 2017):

$$
\begin{gathered}
{\left[\begin{array}{cccc}
Q(k) & * & * & * \\
A_{j} Q(k)+B_{j} Y(k) & Q(k) & * & * \\
\Psi^{1 / 2} Q(k) & 0 & \zeta(k) I & * \\
R^{1 / 2} Y(k) & 0 & 0 & \zeta(k) I
\end{array}\right] \geq 0,} \\
{\left[\begin{array}{cccc}
1 & * & * & * \\
T(k) & Q(k) & * & * \\
\Psi^{1 / 2} \hat{x} & 0 & \zeta(k) I & * \\
R^{1 / 2} u & 0 & 0 & \zeta(k) I
\end{array}\right] \geq 0,} \\
\zeta(k-1)<\zeta(k),
\end{gathered}
$$

onde $Q=Q^{T} \geq 0, F(k)=Y(k) Q^{-1}(k)$ é o ganho do controlador e $T(k)=A_{j} \hat{x}+B_{j} u(k)+L_{o}(y(k)-C \hat{x}(k))$. A obtenção de $L_{o}$ é explicada na seção seguinte.

\subsection{Projeto robusto do observador off-line}

Teorema 1. Projeto do observador off-line (Park et al., 2011): Se existe $G_{e}>0$, e $Y_{e}=G_{e} L_{o}$ satisfazendo a seguinte restrição $L M I$,

$$
\left[\begin{array}{cc}
\rho^{2} G_{e}-L_{e} & * \\
\left(G_{e} A_{j}-Y_{e} C_{j}\right) & G_{e}
\end{array}\right]>0
$$

onde $\rho$ é um parâmetro de projeto e $L_{e}$ é uma matriz peso. Assim o ganho do observador é dado por $L_{o}=G_{e}^{-1} Y_{e}$.

\subsection{Restrição na entrada}

Como proposto por Kothare et al. (1996), Cuzzola et al. (2002) e Park et al. (2011) nós impomos limites à variável de controle. A restrição de entrada $\|u(k+i)\|_{2} \leq u_{j, \max }$, $j=1,2, \ldots, n_{u}, i \geq 0$, é garantido se existir $U, U=U^{T}$ tal que,

$$
\left[\begin{array}{cc}
U_{j j} & * \\
Y^{T} & Q_{j}
\end{array}\right] \geq 0
$$

$\operatorname{com} Q=Q^{T}>0, U_{j j}<u_{j, \max }^{2}, j=1,2, \ldots, n_{u}$.

\section{ALGORITMO OFF-LINE}

Dada uma condição inicial $\left(x_{\text {set }}\right)$ é gerada uma sequência de minimizadores $\zeta_{j}, Q_{j}, U_{j}, Y_{j}$ baseados em (19), (20), (21) e (23). Faça $j:=1$

1. calcular os minimizadores $\zeta_{j}, Q_{j}, U_{j}, Y_{j}$. Armazene $Q_{j}^{-1}, F_{j}$ e $Y_{j}$.

2. se $j<N$ escolha o estado $x_{j+1}$ satisfazendo $\left\|x_{j+1}\right\|_{Q^{-1}}^{2} \leq 1$. Faça $j:=j+1$ e retorne ao passo 1 .

3. calcule $F_{j}=Y_{j} Q_{j}^{-1}$.

4. e aplique a lei de controle $u(k)=-F_{j} \hat{x}(k)+F_{j} v(k)$. 


\section{SIMULAÇÃO NUMÉRICA}

Para testar e comparar a eficácia da técnica off-line MPC, foi usado o modelo do conversor boost descrito na seção 2. Os estados iniciais do sistema (1) e o observador é assumido como $x=[38.461526]^{T}$ e $\hat{x}=[3020]^{T}$, respectivamente. A tensão de referência definida foi de $V_{o}=48 \mathrm{~V}$. O valor máximo do sinal de controle foi $u_{\max }=$ 0.5 e os pontos operacionais do conversor são $380 \mathrm{~W}-$ $1000 \mathrm{~W}$ para um tempo de amostragem de $T_{s}=1 \mathrm{~ms}$ e passo de simulação de $1 \mu s$, foi utilizado $g=h=1$. Assim, o sistema (1) pertence ao seguinte politopo formado pelos quatro modelos discretos locais,

- $f(36 V, 1000 W)$

$$
\begin{gathered}
A_{1}=\left[\begin{array}{cc}
-0.2838 & -7.7479 \\
0.0634 & -0.1137
\end{array}\right], B_{1}=\left[\begin{array}{c}
580.4780 \\
65.2800
\end{array}\right], \\
C_{1}=\left[\begin{array}{ll}
0.0198 & 0.9886
\end{array}\right], D_{1}=-0.7304 .
\end{gathered}
$$

- $f(26 V, 1000 W)$

$$
\begin{gathered}
A_{2}=\left[\begin{array}{cc}
0.0958 & -8.4507 \\
0.0692 & 0.2660
\end{array}\right], B_{2}=\left[\begin{array}{c}
851.9920 \\
53.4470
\end{array}\right], \\
C_{2}=\left[\begin{array}{ll}
0.0143 & 0.9886
\end{array}\right], D_{2}=-1.0054 .
\end{gathered}
$$

- $f(36 \mathrm{~V}, 380 \mathrm{~W})$

$$
\begin{gathered}
A_{3}=\left[\begin{array}{cr}
-0.3102 & -7.9646 \\
0.0652 & -0.1119
\end{array}\right], B_{3}=\left[\begin{array}{c}
542.7340 \\
68.8140
\end{array}\right], \\
C_{3}=\left[\begin{array}{ll}
0.0199 & 0.9956
\end{array}\right], D_{3}=-0.2802 .
\end{gathered}
$$

- $f(26 \mathrm{~V}, 380 \mathrm{~W})$

$$
\begin{gathered}
A_{4}=\left[\begin{array}{ll}
0.0759 & -8.7329 \\
0.0715 & 0.02873
\end{array}\right], B_{4}=\left[\begin{array}{c}
814.2740 \\
58.5880
\end{array}\right], \\
C_{4}=\left[\begin{array}{ll}
0.0144 & 0.9956
\end{array}\right], D_{4}=-0.3871 .
\end{gathered}
$$

As matrizes de ponderação são

$$
\Psi=\left[\begin{array}{lll}
1 & 0 & 0 \\
0 & 1 & 0 \\
0 & 0 & 1
\end{array}\right], L_{e}=\left[\begin{array}{ll}
1 & 0 \\
0 & 1
\end{array}\right] \text { e } \quad R=0.1 .
$$

O ganho do observador de estado $L_{o}$ obtido foi,

$$
L_{o}=\left[\begin{array}{lll}
-8.299106 & 0.077623
\end{array}\right]^{T},
$$

com $\rho=0.6^{1 / 2}$. O ganho do observador foi o mesmo para o algoritmo MPC online e off-line.

O ganho MPC $F$ para ambos os algoritmos online $\left(F_{o n}\right)$ e off-line $\left(F_{\text {off }}\right)$ obtidos foram,

$$
\begin{gathered}
F_{\text {on }}=[\underbrace{0.000123-0.007411}_{K} \underbrace{-0.001032}_{K}] \\
F_{\text {off }}=[\underbrace{0.000030-0.003830}_{K_{i}} \underbrace{-0.001488}_{K_{i}}] .
\end{gathered}
$$

O ganho obtido no MPC online $\left(F_{\text {on }}\right)$ não é estático, isto é, o ganho varia a cada iteração. Deste modo, $F_{\text {on }}$ é o ganho obtido na primeira iteração do algoritmo online proposto por Park et al. (2011). Já o ganho $F_{\text {off }}$ é o ganho obtido com o algoritmo off-line proposto neste trabalho.

Uma variação da tensão de entrada $26 \mathrm{~V}-36 \mathrm{~V}$ foi realizada no instante de $0.075 \mathrm{~s}$, cujo intervalo de análise foi entre $0 s-0.15 s$.

Na Figura 3 são mostradas as elipsoides de estabilidade definidos por $Q^{-1}$ com 10 pontos discretos $\left(x_{\text {set }}=[48.0000\right.$ $\begin{array}{llllll}-3.6942 & -23.6553 & 11.3360 & 7.8756 & -9.0638 & -0.5195 \\ 4.9506 & -\end{array}$ $1.6958-1.9171])$ obtidos através do algoritmo MPC offline. $Q^{-1}$ assume um comportamento elipsoidal no plano geométrico e sua estabilidade robusta é garantida com o conjunto $x_{\text {set }}$ em regime permanente pois as elipse convergem para origem. Quanto mais próxima da origem estive a elipse mais estável será a resposta do sistema. Os estados $x_{1}$ e $x_{2}$ são respectivamente os pontos discretos da corrente do indutor $\left(i_{L}\right)$ e tensão no capacitor $\left(V_{c}\right)$.

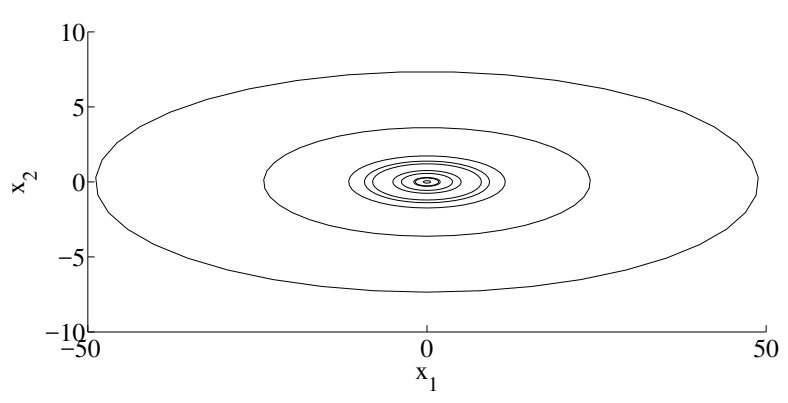

Figura 3. Elipsóides de estabilidade.

A Figura 4 mostra a tensão de saída $\left(V_{o}\right),(-)$ é a saída do MPC online e (+) a saída do MPC off-line. Ambos os controladores seguiram a tensão de referência predefinida. A diferença entre os sinais é mínima, no entanto, é possível ver que o controlador online forneceu uma resposta um pouco mais rápida, mas com um maior overshoot.

Na Figura 5 é mostrado o sinal de controle $u(k)$. É possível ver que, para ambas as técnicas de controle, a restrição foi satisfeita. Apesar de uma resposta mais lenta obtida com o off-line.

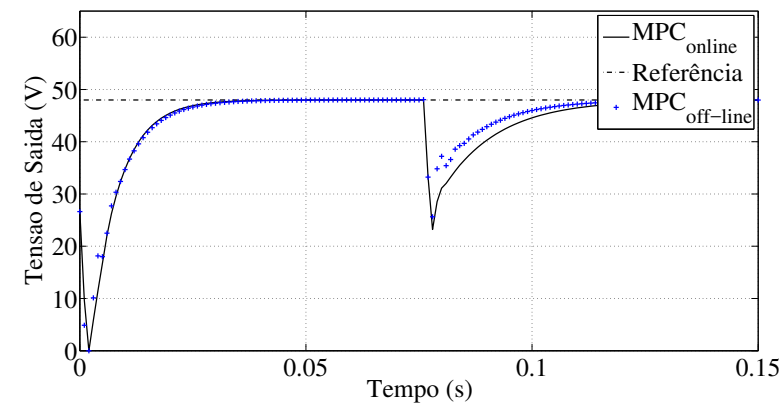

Figura 4. Tensão de saída $\left(V_{o}\right)$, (-) é a saída online e $(+)$ a off-line.

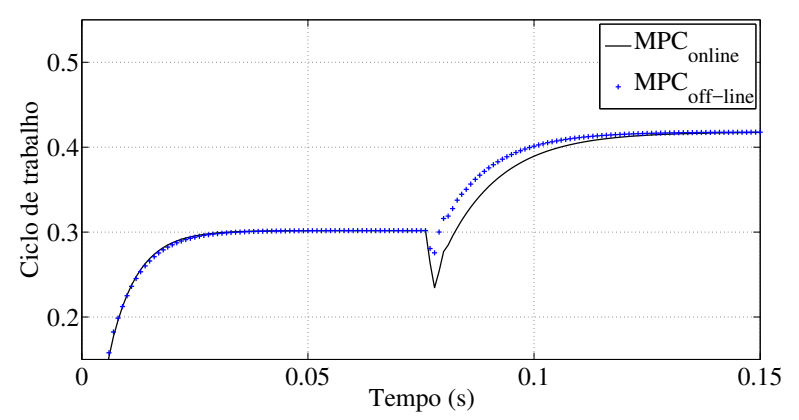

Figura 5. Sinal de controle $(u),(-)$ é a saída online e $(+)$ a off-line. 
As Figuras 6 e 7 mostram a corrente do indutor $\left(i_{L}\right)$ e a corrente de saída $\left(i_{o}\right)$ respectivamente.

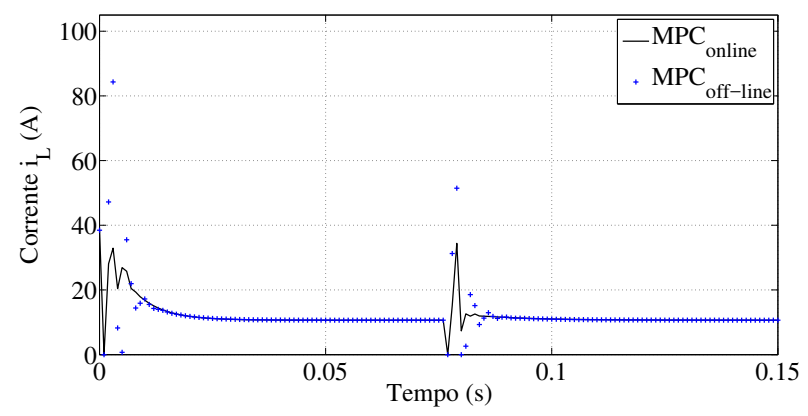

Figura 6. Corrente no indutor $\left(i_{L}\right),(-)$ é a saída online e $(+)$ o off-line.

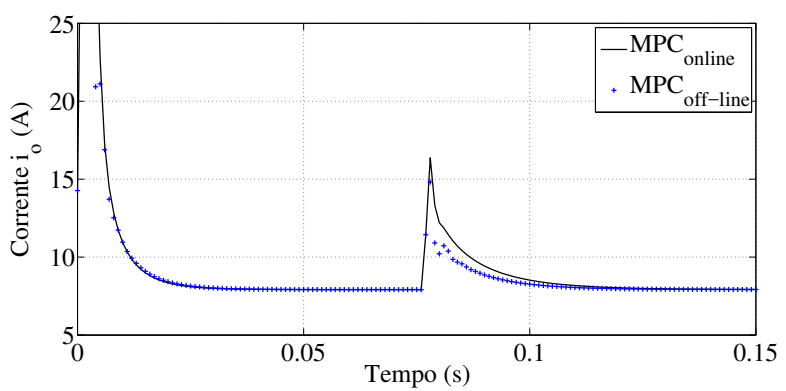

Figura 7. Corrente na saída $\left(i_{o}\right),(-)$ é a saída online e $(+)$ o off-line.

Na Figura 8 temos a corrente estimada do indutor $\left(i_{L}\right)$ online e off-line, a linha azul $(+)$ é a saída do observado $\hat{x}(k)$ e a linha preta $(-)$ é $x(k)$. As correntes estimadas no processo online apresentaram um resultado relativamente superior ao obtido no processo off-line, mas com overshoot.

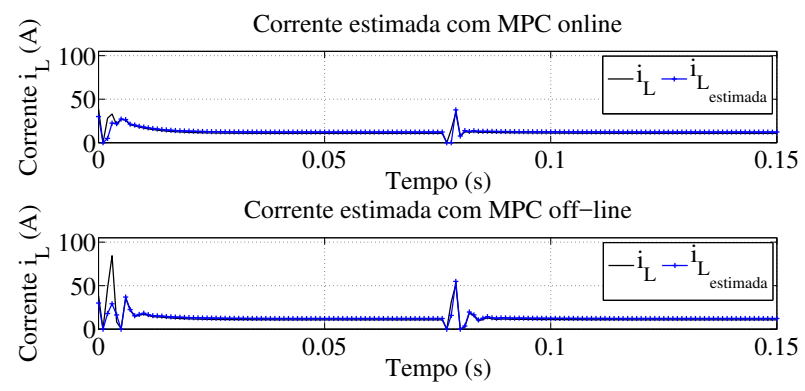

Figura 8. Corrente estimada no indutor $\left(i_{L}\right),(+)$ saída do observado.

\section{CONCLUSÃO}

Os resultados foram apresentados entre os processos online e off-line iterativo. Foi visto que o processo iterativo offline garante os mesmos princípios da estabilidade assintótica robusta do processo online. A vantagem de usar o processo off-line é que esse método não requer tanto processamento quanto o método online. Assim, pode ser facilmente aplicado em uma planta experimental como por exemplo no conversor boost. Como trabalho futuro, os autores pretendem realizar a simulação experimental no conversor e realizar o relaxamento da LMI do controlador.

\section{REFERÊNCIAS}

Aguirre, L.A., Bruciapaglia, A.H., Miyagi, P.E., e Piqueira, J.R.C. (2007). Enciclopédia de automática: controle e automação. Blucher.

Amirifar, R. (2005). Extended dynamic matrix control design for a dc-dc power converter. In System Theory, 2005. SSST'05. Proceedings of the Thirty-Seventh Southeastern Symposium on, 191-195. IEEE.

Bascopé, G.T. e Barbi, I. (2000). Generation of a family of non-isolated dc-dc pwm converters using new threestate switching cells. In Power Electronics Specialists Conference, 2000. PESC 00. 2000 IEEE 31st Annual, volume 2, 858-863. IEEE.

Camacho, E.F. e Alba, C.B. (2013). Model predictive control. Springer Science \& Business Media.

Cheng, L., Acuna, P., Aguilera, R.P., Jiang, J., Wei, S., Fletcher, J., e Lu, D.D.C. (2017). Model predictive control for dc-dc boost converters with reduced-prediction horizon and constant switching frequency. IEEE Transactions on Power Electronics.

Costa, M.V.S. (2012). Controladores Robustos D-LQI e D-Alocação de polos Otimizados via LMI Aplicados a um Conversor Boost Alto Ganho com Célula de Comutação Três Estados. Mestrado em engenharia elétrica, Universidade Federal do Ceará, Fortaleza. $120 \mathrm{f}$.

Costa, M.V.S. (2017). Controle MPC robusto aplicado ao conversor boost CCTE otimizado por inequações matriciais lineares. Doutorado em engenharia elétrica, Universidade Federal do Ceará, Fortaleza.

Costa, M.V., Reis, F.E., Campos, J.C., et al. (2017). Controlador robusto mpc-lmi aplicado ao conversor boost com célula de comutação de três estados. Eletrônica de Potência, Campo Grande, 81-90.

Cuzzola, F.A., Geromel, J.C., e Morari, M. (2002). An improved approach for constrained robust model predictive control. Automatica, 38(7), 1183-1189.

Guldemir, H. (2011). Modeling and sliding mode control of dc-dc buck-boost converter. In Proc. 6th Int. advanced technological Symp, volume 4, 475-480.

$\mathrm{Hu}$, J. e Ding, B. (2019). An efficient offline implementation for output feedback min-max mpc. International Journal of Robust and Nonlinear Control, 29(2), 492506.

Kim, T.H. e Lee, H.W. (2017). Quasi-min-max outputfeedback model predictive control for lpv systems with input saturation. International Journal of Control, Automation and Systems, 15(3), 1069-1076.

Kothare, M.V., Balakrishnan, V., e Morari, M. (1996). Robust constrained model predictive control using linear matrix inequalities. Automatica, 32(10), 1361-1379.

Linares-Flores, J., Mendez, A.H., Garcia-Rodriguez, C., e Sira-Ramirez, H. (2014). Robust nonlinear adaptive control of a boost converter via algebraic parameter identification. IEEE Transactions on Industrial Electronics, 61(8), 4105-4114.

Longge, Z. e Yan, Y. (2017). Robust shrinking ellipsoid model predictive control for linear parameter varying system. PLOS ONE, 12(6), e0178625. doi:10.1371/ journal.pone.0178625.

Lorenzen, M., Dabbene, F., Tempo, R., e Allgöwer, F. (2017). Stochastic mpc with offline uncertainty sampling. Automatica, 81, 176-183. 
Middlebrook, R. e Cuk, S. (1976). A general unified approach to modelling switching-converter power stages. In Power Electronics Specialists Conference, 1976 IEEE, 18-34. IEEE.

Moradi, S.M., Akbari, A., e Mirzaei, M. (2018). An offline lmi-based robust model predictive control of vehicle active suspension system with parameter uncertainty. Transactions of the Institute of Measurement and Control.

Olalla, C., Leyva, R., Queinnec, I., e Maksimovic, D. (2012). Robust gain-scheduled control of switchedmode dc-dc converters. IEEE Transactions on Power Electronics, 27(6), 3006-3019.

Park, J.H., Kim, T.H., e Sugie, T. (2011). Output feedback model predictive control for lpv systems based on quasimin-max algorithm. Automatica, 47(9), 2052-2058.

Paulson, J.A., Xie, L., e Mesbah, A. (2017). Offsetfree robust mpc of systems with mixed stochastic and deterministic uncertainty. IFAC-PapersOnLine, 50(1), 3530-3535.

Ping, X. (2017). Output feedback robust mpc based on offline observer for lpv systems via quadratic boundedness. Asian Journal of Control, 19(4), 1641-1653.

Rego, R.C.B., Costa, M.V.S., Reis, F.E.U., e Bascopé, R.P.T. (2018). Análise e simulação do controlador mpc-aw-lmi aplicado ao conversor ccte operando em condições de saturação no sinal de controle. Congresso Brasileiro de Automática, XXII.

Wada, N., Saito, K., e Saeki, M. (2006). Model predictive control for linear parameter varying systems using parameter dependent lyapunov function. IEEE Transactions on Circuits and Systems II: Express Briefs, 53(12), 1446-1450. doi:10.1109/tcsii.2006.883832.

Wan, Z. e Kothare, M.V. (2002). Robust output feedback model predictive control using off-line linear matrix inequalities. Journal of Process Control, 12(7), 763-774.

Wan, Z. e Kothare, M.V. (2003). An efficient off-line formulation of robust model predictive control using linear matrix inequalities. Automatica, 39(5), 837-846.

Zheng, H., Zou, T., Hu, J., e Yu, H. (2018). An offline optimization and online table look-up strategy of twolayer model predictive control. IEEE Access, 6, 4743347441. 